University of Nebraska - Lincoln

DigitalCommons@University of Nebraska - Lincoln

2008

\title{
Bilateral Claudication Results in Alterations in the Gait Biomechanics at the Hip and Ankle Joints
}

\author{
Shing-Jye Chen \\ University of Nebraska at Omaha \\ Iraklis Pipinos \\ University of Nebraska Medical Center, Omaha, NE, USA, ipipinos@unmc.edu \\ Jason Johanning \\ University of Nebraska Medical Center, Omaha, NE, USA, jjohanning@unmc.edu \\ Matija Radovic \\ University of Nebraska at Omaha \\ Jessie M. Huisinga \\ University of Nebraska at Omaha
}

See next page for additional authors

Follow this and additional works at: https://digitalcommons.unl.edu/publichealthresources

Digitalrt of the Public Health Commons

Commons

Network Shing-Jye; Pipinos, Iraklis; Johanning, Jason; Radovic, Matija; Huisinga, Jessie M.; Myers, Sara A.; åg Stergiou, Nikolaos, "Bilateral Claudication Results in Alterations in the Gait Biomechanics at the Hip and Ankle Joints" (2008). Public Health Resources. 3.

https://digitalcommons.unl.edu/publichealthresources/3

This Article is brought to you for free and open access by the Public Health Resources at DigitalCommons@University of Nebraska - Lincoln. It has been accepted for inclusion in Public Health Resources by an authorized administrator of DigitalCommons@University of Nebraska - Lincoln. 


\section{Authors}

Shing-Jye Chen, Iraklis Pipinos, Jason Johanning, Matija Radovic, Jessie M. Huisinga, Sara A. Myers, and Nikolaos Stergiou 


\title{
Bilateral claudication results in alterations in the gait biomechanics at the hip and ankle joints
}

\author{
Shing-Jye Chen ${ }^{\mathrm{a}, *}$, Iraklis Pipinos ${ }^{\mathrm{b}, \mathrm{c}}$, Jason Johanning ${ }^{\mathrm{b}, \mathrm{c}}$, Matija Radovic ${ }^{\mathrm{a}}$, \\ Jessie M. Huisinga ${ }^{\mathrm{a}}$, Sara A. Myers ${ }^{\mathrm{a}}$, Nick Stergiou ${ }^{\mathrm{a}, \mathrm{b}}$ \\ ${ }^{a}$ HPER Biomechanics Laboratory, School of Health, Physical Education \& Recreation, University of Nebraska at Omaha, \\ 6001 Dodge Street, Omaha, NE 68182-0216, USA \\ ${ }^{\mathrm{b}}$ University of Nebraska Medical Center, Omaha, NE, USA \\ ${ }^{\mathrm{c}}$ Veterans Affairs Medical Center, Omaha, NE, USA
}

Accepted 8 May 2008

\begin{abstract}
Claudication is the most common symptomatic manifestation of peripheral arterial disease (PAD), producing significant ambulatory compromise. The purpose of this study was to use advanced biomechanical gait analysis to determine the gait alterations occurring in claudicating patients both before and after onset of claudication pain in their legs. Hip, knee, and ankle joint moments were measured in claudicating patients (age: $64.46 \pm 8.47$ years; body mass: $80.70 \pm 12.64 \mathrm{~kg}$; body height: $1.72 \pm 0.08 \mathrm{~m}$ ) and were compared to gender-age-body mass-height-matched healthy controls (age 66.27 \pm 9.22 years; body mass: $77.89 \pm 10.65 \mathrm{~kg}$; body height: $1.74 \pm 0.08 \mathrm{~m}$ ). The claudicating patients were evaluated both before (pain-free (PF) condition) and after (pain condition) onset of claudication pain in their legs. Thirteen symptomatic PAD patients (26 claudicating limbs) with bilateral intermittent claudication (IC) and 11 healthy controls ( 22 control limbs) were tested during level walking at their self-selected speed. Compared to controls, PAD hip and ankle joints demonstrated significant angular kinematics and net internal moment changes. Alterations were present both in PF and pain conditions with several of them becoming worse in the pain condition. Both PF and pain conditions resulted in significantly reduced peak hip extensor moment $(5.62 \pm 1.40$ and $5.63 \pm 1.33 \% \mathrm{BW} \times \mathrm{BH}$, respectively) during early stance as compared to controls $(7.53 \pm 1.16 \% \mathrm{BW} \times \mathrm{BH})$. In the pain condition, PAD patients had a significantly reduced ankle plantar flexor moment $(7.56 \pm 1.41 \%$ $\mathrm{BW} \times \mathrm{BH})$ during late stance as compared to controls $(8.65 \pm 1.27 \% \mathrm{BW} \times \mathrm{BH})$. Furthermore, when comparing $\mathrm{PF}$ to pain conditions, there was a decreased peak plantar flexor moment (PF condition: $8.23 \pm 1.37$ vs. pain condition: $7.56 \pm 1.41 \% \mathrm{BW} \times \mathrm{BH}$ ) during late stance. The findings point to a weakness in the posterior compartment muscles of the hip and calf as being the key factor underlying the PAD gait adaptations.

Our findings establish a detailed baseline description of the changes present in PAD patient's joint angles and moments during walking. Since IC is primarily a gait disability, better understanding of the abnormalities in joint and muscle function will enhance our understanding of the gait impairment and may lead to novel, gait-specific treatments.
\end{abstract}

Keywords: Peripheral arterial disease; Claudication; Joint moment; Biomechanics; Gait

\section{Introduction}

Intermittent claudication (IC) is the most common symptomatic manifestation of peripheral arterial disease (PAD) presenting as ischemic leg muscle pain and gait

\footnotetext{
*Corresponding author. Tel.: + 1402554 3246; fax: + 14025543693.

E-mail address: shingjychen@mail.unomaha.edu (S.-J. Chen).
}

dysfunction. Claudication pain is the result of exerciseinduced reduction in blood supply to the working muscle of the lower extremity (Meru et al., 2006). This ischemic pain is induced by physical activity (i.e. walking) and relieved during rest. IC and its associated ambulatory dysfunction are associated with poor health outcomes, physical dependence (Atkins and Gardner, 2004; Gardner and Clancy, 2006) and impaired quality of life (Liles et al., 
2006; Menard et al., 2004). Currently, baseline ambulatory impairment and success of treatment (e.g., supervised treadmill based exercise, angioplasty, bypass) are evaluated using limited clinical (resting Ankle-Brachial Index (ABI), walking distance evaluation) (Oka et al., 2005) and rudimentary time-distance tools (Gardner et al., 2001; McDermott et al., 2001). Previous work has demonstrated that PAD patients walk slower with a decreased cadence, increased stance time, shorter stride length and a narrower step width as compared with controls (Gardner et al., 2001; McDermott et al., 2001). However, these changes are unable to describe in detail the specific physical impairments of claudicating patients and in our understanding of its underlying pathophysiology.

Detailed quantitative evaluation of gait can be provided using advanced biomechanical analysis with joint kinetics and kinematics (Fosang and Baker, 2006; Winter, 2005). This analysis has been used extensively to characterize gait abnormalities of patients with cerebral palsy (Chang et al., 2006), stroke (McGinley et al., 2006; Yavuzer et al., 2006), head injury (Basford et al., 2003; Vallée et al., 2006) and other neuromuscular disorders (Barker et al., 2006; Runge and Hunter, 2006). Furthermore, advanced biomechanical analysis has been used to guide rehabilitation and surgical treatment (Baker, 2006; Jöbges et al., 2004) and assess therapeutic outcomes (Georgoulis et al., 2007; Stergiou et al., 2007). Therefore, the purpose of the present study was to utilize advanced biomechanical analysis in order to determine the gait impairment of claudicating PAD patients. Joint kinetic and kinematic parameters were evaluated in PAD patients before and after the onset of claudication pain while walking in pain-free (PF) and pain (P) conditions, respectively. The PAD patients were compared to gender-, height-, mass-, and age-matched healthy controls. We also explored the relationships between ABI, the Walking Impairment Questionnaire (WIQ) subscale scores, and peak joint moments while walking. We hypothesized that as patients experienced pain due to lack of blood flow in the claudicating limbs, the gait parameters would be significantly altered as compared to baseline ambulation and healthy controls. We also hypothesized that the resting ABI and WIQ scores would positively correlate with the peak joint moments.

\section{Methods}

\subsection{Subject inclusion and exclusion criteria}

Thirteen male and female symptomatic PAD patients (age: $64.46 \pm 8.47$ years, body mass: $80.70 \pm 12.64 \mathrm{~kg}$; body height: $1.72 \pm 0.08 \mathrm{~m}$ ) diagnosed with moderate arterial occlusive disease and bilateral claudication were recruited from the vascular surgery clinics of the local Medical Centers. In addition, 11 gender-, age-, body mass-, and height-matched healthy controls (age: $66.27 \pm 9.22$ years, body mass: $77.89 \pm 10.65 \mathrm{~kg}$; body height: $1.74 \pm 0.08 \mathrm{~m}$ ) volunteered to participate. Patients and controls were screened and evaluated by two board certified vascular surgeons. Those PAD patients with ambulation limiting cardiac, pulmonary, neuromuscular, or musculoskeletal disease or those who experienced pain or discomfort during walking for any reason other than claudication (i.e. arthritis, low back pain, musculoskeletal problems, neuropathy) were excluded. Patient evaluation included resting ABI (a measurement below 0.90 was present in all subjects with claudication), detailed history, physical exam, and direct assessment/observation of the patient's walking impairment. A vascular surgeon observed the patient walking and recorded all symptoms and signs affecting ambulation to ensure limitation was secondary to claudication pain.

Control subjects had an ABI greater than 0.90 and no subjective or objective ambulatory dysfunction. Controls were screened in a similar fashion as PAD patients and were excluded for the same ambulation limiting problems or if pain was experienced during walking. Informed consent was obtained from all subjects prior to data collection according to the guidelines of the University's Institutional Review Board. The gait of all recruited participants was tested in the Biomechanics Laboratory. In addition to gait analysis, the self-administered modified WIQ (Coyne et al., 2003) including the four subscales of (a) pain severity, (b) walking distance, (c) speed, and (d) stair climbing was obtained from all recruited patients.

\subsection{Experimental procedure and data collection}

Prior to data collection, reflective markers were placed at specific anatomical locations of each subject's lower limb utilizing the modified Helen Hayes marker set (Houck et al., 2004). Each subject was directed to walk using their self-selected pace over a $10 \mathrm{~m}$ pathway, while threedimensional (3D) marker trajectories and ground reaction force data were simultaneously collected. The 3D marker trajectories were captured with a six high-speed real-time camera system (EvaRT 5.0, Motion Analysis Corporation, Santa Rosa, CA) sampling at $60 \mathrm{~Hz}$. The ground reaction force data were acquired with a Kistler force platform sampling at $600 \mathrm{~Hz}$.

Each patient was tested in the "PF" condition and then in the pain (P)induced condition. During PF testing, mandatory rest occurred between walking trials to ensure that all trials were in a PF condition. Once patients completed all PF trials, P trials were performed. In order to accomplish this, a common clinical protocol was used where each patient was asked to walk on an inclined treadmill with $10 \%$ grade at a speed of $1.5 \mathrm{mph}$ (DiBianco et al., 1984) until claudication pain was established. The patients were then immediately removed from the treadmill and returned to the collection walk-way to acquire the data for the $\mathrm{P}$ condition without the mandatory resting periods. Controls completed only the PF condition trials. A total of five successful walking trials was collected from each leg of the subjects for each condition. A successful walking trial was determined by the subject's foot being completely within the force platform.

\subsection{Data analysis}

Peak values of the joint kinetics and kinematics measured in the sagittal plane during the early and late stance phases were analyzed (Figs. 1 and 2). Time-distance parameters were also analyzed from one complete stride of each subject and for each testing condition. A low-pass fourth-order Butterworth filter with a $6 \mathrm{~Hz}$ cutoff was used to smooth the marker trajectories during post-data processing. The OrthoTrak 6.29 software package (Motion Analysis Corporation, Santa Rosa, CA) was used to calculate the time-distance parameters, the joint kinetics, and kinematics of each subject. The joint kinetics was scaled to body weight and body height.

\subsection{Statistical analysis}

Group means of the peak joint kinetics, peak joint kinematics, and the time-distance parameters were calculated for each testing condition (PF and $\mathrm{P}$ conditions) by combining all legs of each group. Thus, an $N$ of 26 limbs was generated for the PAD group and an $N$ of 22 limbs for the 

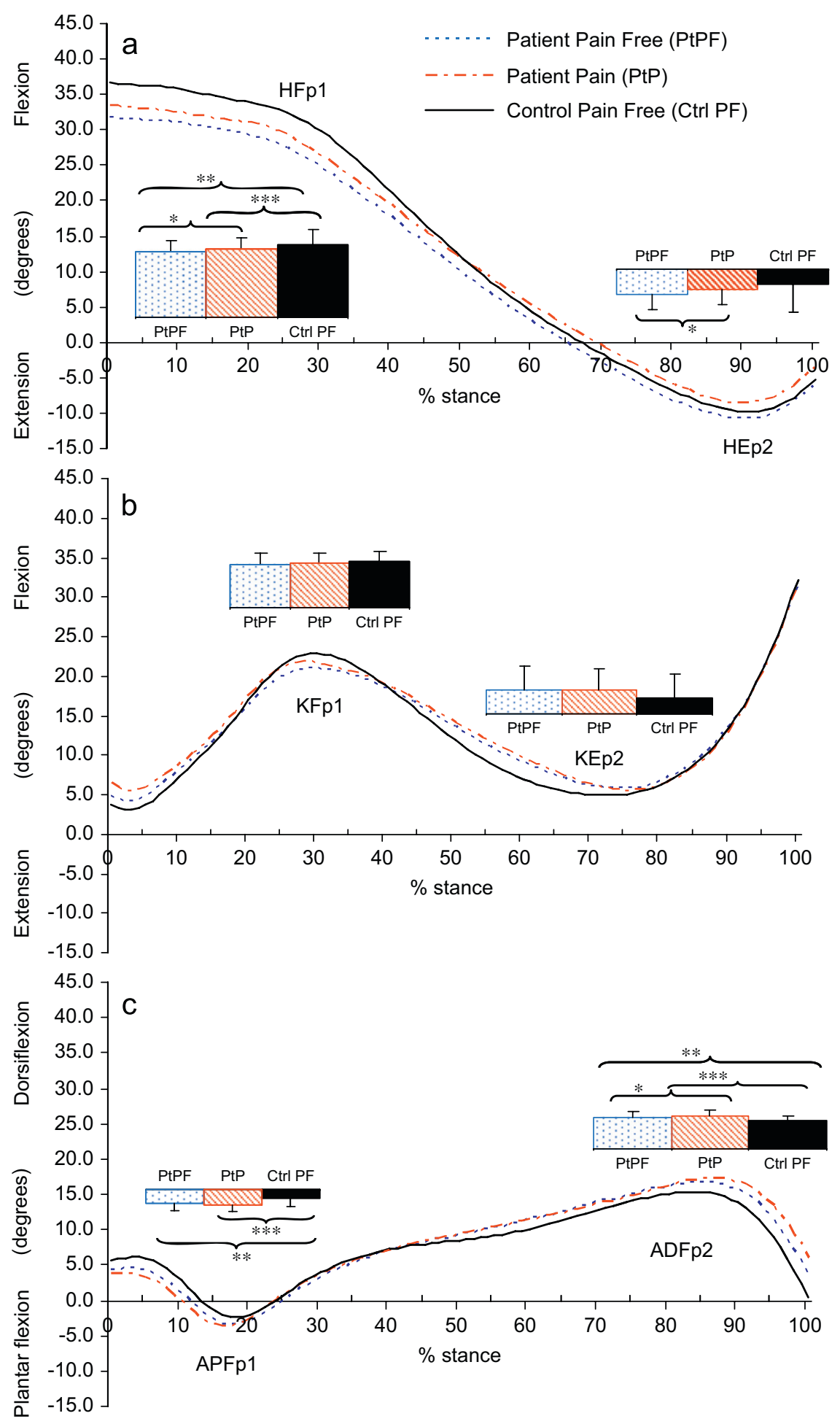

Fig. 1. The ensemble-average flexion and extension of both supporting limbs for the PAD patients (Pt: pain free and pain; $N=26$ limbs) and the controls (Ctrl: $N=22$ limbs) during the stance phase for the (a) hip, (b) knee, and (c) ankle joints. Note: HFp1/HEp2: hip flexion/extension peak 1 and peak 2 during the early and late stance phases, respectively. KFp1/KEp2: knee flexion/extension peak 1 and peak 2 during the early and late stance phases, respectively. APFp1/ADFp2: ankle plantar flexion/dorsiflexion peak 1 and peak 2 during the early and late stance phases, respectively. ${ }^{*} p<0.05$, significant differences between testing conditions (PF vs. P). ${ }^{* *} p<0.05$, significant differences between groups (patient PF condition vs. control PF). $* * * p<0.05$, significant differences between groups (patient $\mathrm{P}$ condition vs. control PF).

control group. Paired $t$-tests were used to detect the effects of induced claudication pain, and independent $t$-tests were used to examine group effects for each dependent variable. Pearson correlations were performed to evaluate the relationships between the patients' ABIs, the peak joint moments, and the WIQ four subscales in the PF and P conditions. The level of significance was set at 0.05 . Parametric statistics were utilized because gait analysis data have been shown to demonstrate normality (Benedetti et al., 1998). 

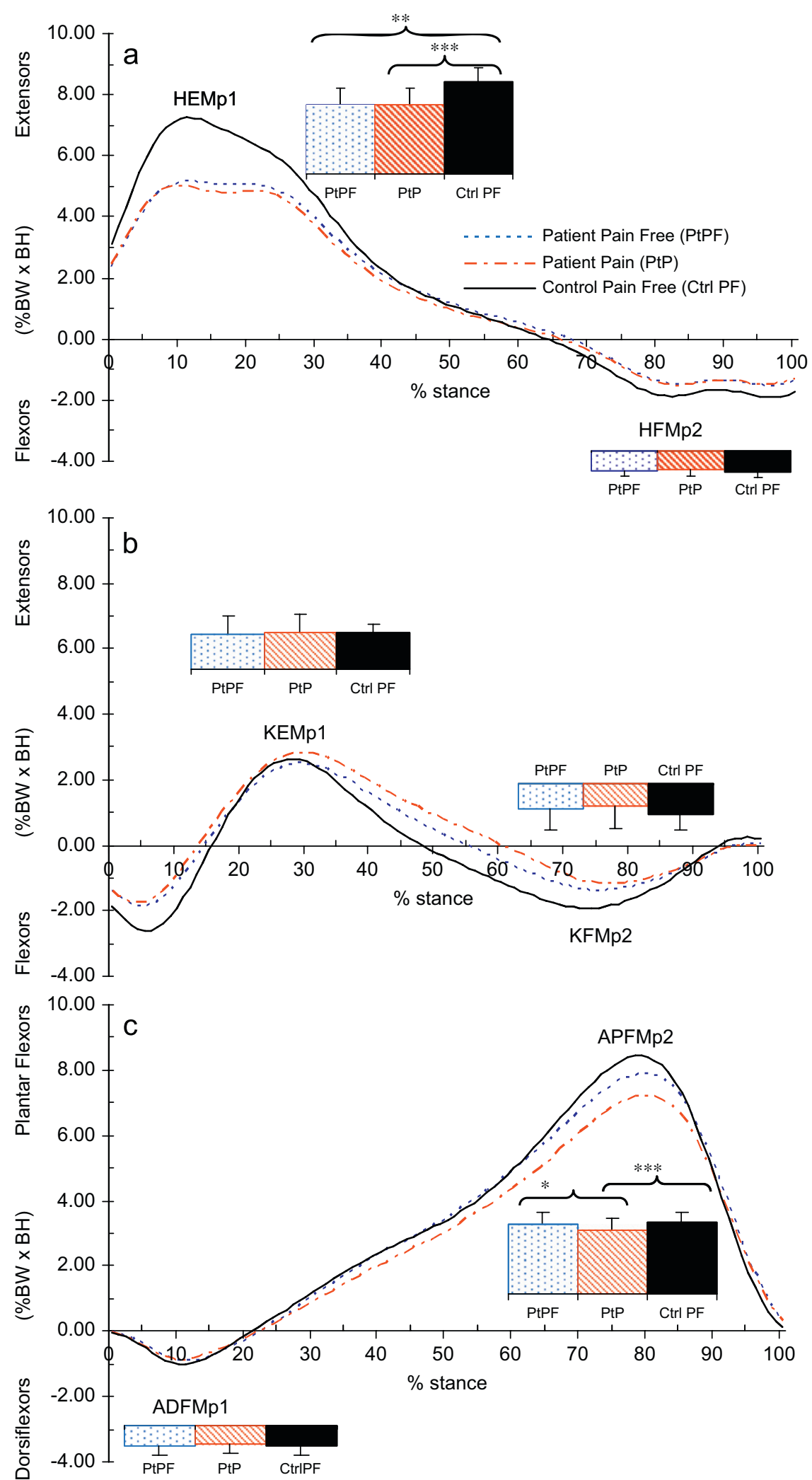

Fig. 2. The ensemble-average net extensor/flexor moment curves of both supporting limbs for the patients (Pt: pain free and pain; $N=26$ limbs) and the controls (Ctrl: $N=22$ limbs) during the stance phase for the (a) hip, (b) knee, and (c) ankle joints. A positive (+) value indicates a net internal extensor/plantar flexor moment, and a negative (-) value indicates a net internal flexor/dorsiflexor moment. Moments are normalized to body weight (BW) and body height (BH). Note: HEM p1/HFM p2: hip extension/flexion moment peak 1 and peak 2 during the early and late stance phases, respectively. KEM p1/KFM p2: knee extension/ flexion moment peak 1 and peak 2 during the early and late stance phases, respectively. ADFM p1/APFM p2: ankle dorsiflexion/plantar flexion moment peak 1 and peak 2 during the early and late stance phases, respectively. ${ }^{*} p<0.05$, significant differences between testing conditions (PF vs. P). ${ }^{* *} p<0.05$, significant differences between groups (patient PF condition vs. control PF). ${ }^{* *} p<0.05$, significant differences between groups (patient $\mathrm{P}$ condition vs. control PF). 


\section{Results}

\subsection{Time-distance gait measurements}

The baseline clinical characteristics of the patients $(N=26$ limbs $)$ and the healthy controls $(N=22$ limbs $)$ are presented in Table 1 . When compared to controls, patients showed significant decreases in gait velocity, stride length, and cadence and a significant increase in double support time in the PF condition (Table 2). The differences for the same parameters were further amplified when the $\mathrm{P}$ condition was compared with the controls. Additional significant differences were also present with increased stance phase, increased double support phase, and decreased swing phase (Table 2). No significant differences

Table 1

Baseline characteristics of bilateral claudicating PAD patients and healthy control subjects

\begin{tabular}{lll}
\hline Clinical characteristics & Patient (26 limbs) & Control (22 limbs) \\
\hline Gender (male/female) & $12 / 1$ & $10 / 1$ \\
Age (years) & $64.46 \pm 8.47$ & $66.27 \pm 9.22$ \\
Body mass (kg) & $80.70 \pm 12.64$ & $77.89 \pm 10.65$ \\
Body height (m) & $1.72 \pm 0.08$ & $1.74 \pm 0.08$ \\
Disease duration (years) & $6.25 \pm 3.84$ & 0 \\
ABI & & \\
$\quad$ Right limb & $0.49 \pm 0.16$ & $1.1 \pm 0.11$ \\
Left limb & $0.56 \pm 0.17$ & $1.1 \pm 0.09$ \\
Smokers, $n(\%)$ & $0(0)$ & $0(0)$ \\
Hypertension, $n(\%)$ & $11(84.6)$ & $0(0)$ \\
Diabetes mellitus, $n(\%)$ & $0(0)$ & $0(0)$ \\
Hyperlipidemia, $n(\%)$ & $12(92.3)$ & $0(0)$ \\
BMI & $27.16 \pm 4.51$ & $25.60 \pm 2.94$ \\
WIQ domains & & \\
Claudication pain & $57.69 \pm 15.76$ & $\mathrm{n} / \mathrm{a}$ \\
Walking distance & $47.37 \pm 23.29$ & $\mathrm{n} / \mathrm{a}$ \\
Walking speed & $47.44 \pm 28.31$ & $\mathrm{n} / \mathrm{a}$ \\
Stair climbing & $52.57 \pm 25.77$ & $\mathrm{n} / \mathrm{a}$ \\
\hline
\end{tabular}

Note: ABI: Ankle-Brachial Index; BMI: Body Mass Index; WIQ: Walking Impairment Questionnaires; $n$ : a number of subjects. were found for step width. Comparing the PF to $\mathrm{P}$ conditions, there was a significant decrease in the gait velocity and stride length (Table 2).

\subsection{Joint angles}

Several changes were noted both in the early stance (after heel contact or braking phase) and the late stance (before toe off or propulsion phase). In comparison with controls, patients in the PF condition showed a significantly decreased hip peak flexion $\left(34.75^{\circ} \pm 7.1\right.$ vs. $30.95^{\circ} \pm 5.20$, respectively) during the early stance, a significantly increased peak ankle plantar flexion $\left(2.43^{\circ} \pm 2.11\right.$ vs. $3.63^{\circ} \pm 1.86$, respectively) during the early stance, and a significantly increased peak ankle dorsiflexion $\left(15.09^{\circ} \pm 3.01\right.$ vs. $17.14^{\circ} \pm 2.89$, respectively) during late stance. Once in the $\mathrm{P}$ condition, the results for the same parameters remained significantly different compared to healthy controls (Fig. 1). Furthermore when comparing PF to $\mathrm{P}$ conditions, there was significantly increased peak hip flexion (PF: $30.95^{\circ} \pm 5.20$ vs. P: $32.41^{\circ} \pm 5.32$ ) at the early stance and significantly decreased peak hip extension at late stance for the patients (Fig. 1). In addition, the $\mathrm{P}$ condition resulted in a significantly increased peak ankle dorsiflexion during the late stance.

\subsection{Joint moments}

Patients in the PF condition had a significantly reduced peak hip extensor moment $(5.62 \pm 1.40 \% \mathrm{BW} \times \mathrm{BH})$ during early stance as compared to controls $(7.53 \pm 1.16 \%$ $\mathrm{BW} \times \mathrm{BH}$ ) (Fig. 2a). In the $\mathrm{P}$ condition, patients were found to have a significantly reduced peak hip extensor moment $(5.63 \pm 1.33 \% \mathrm{BW} \times \mathrm{BH})$ during early stance and a significantly reduced ankle plantar flexor moment $(7.56 \pm 1.41 \% \mathrm{BW} \times \mathrm{BH})$ during late stance as compared to controls (7.53 \pm 1.16 and $8.65 \pm 1.27$, respectively; Fig. 2a and c). Furthermore, when comparing $\mathrm{PF}$ to $\mathrm{P}$, there was a decreased peak plantar flexor moment (PF: $8.23 \pm 1.37$ vs. P: $7.56 \pm 1.41$ ) during the late stance (Fig. 2c).

Table 2

Time-distance gait measurements for both patient and control groups

\begin{tabular}{|c|c|c|c|}
\hline & \multicolumn{2}{|c|}{ Patient $(N=26$ limbs $)$} & \multirow{2}{*}{$\frac{\text { Control }(N=22 \text { limbs })}{\text { Pain free }(\mathrm{PF})}$} \\
\hline & Pain free $(\mathrm{PF})$ & Pain $(\mathrm{P})$ & \\
\hline Stride length $(\mathrm{m})$ & $1.34 \pm 0.11^{*, * *}$ & $1.28 \pm 0.10^{* * *}$ & $1.52 \pm 0.10$ \\
\hline Cadence (steps/min) & $102.19 \pm 8.49^{* *}$ & $100.71 \pm 7.36^{* * *}$ & $108.34 \pm 9.05$ \\
\hline Step width $(\mathrm{m})$ & $0.14 \pm 0.04$ & $0.15 \pm 0.03$ & $1.32 \pm 0.03$ \\
\hline Double support (\%) & $11.87 \pm 1.95^{* *}$ & $12.38 \pm 2.52^{* * *}$ & $10.21 \pm 1.97$ \\
\hline
\end{tabular}

${ }^{*} p<0.05$, significant differences between testing conditions (PF vs. P).

$* * p<0.05$, significant differences between groups (patient PF condition vs. control PF)

${ }^{* * *} p<0.05$, significant differences between groups (patient $\mathrm{P}$ condition vs. control PF). 
Table 3

Pearson correlations $(r)$ among the peak joint moments at hip, knee, and ankle during walking without pain (PF) and with pain (P) claudication conditions, resting ABI, and the four WIQ subscales for pain, walking distance, speed, and stairs

\begin{tabular}{|c|c|c|c|c|c|c|c|c|c|c|c|}
\hline Pain condition & ABI & WIQ pain & WIQ distance & WIQ speed & WIQ stairs & ADFM p1 & APFM p2 & KEM pl & KFM p2 & HEM pl & HFM p2 \\
\hline \multicolumn{12}{|l|}{ Pain free $(\mathrm{PF})$} \\
\hline $\mathrm{ABI}$ & & 0.476 & $0.67^{*}$ & 0.45 & 0.21 & 0.44 & -.17 & 0.25 & 0.09 & 0.19 & 0.05 \\
\hline WIQ: pain & & & $0.59^{*}$ & $0.57^{*}$ & 0.24 & -0.13 & -0.075 & $0.71^{*}$ & $0.66^{*}$ & 0.00 & -0.32 \\
\hline WIQ: distance & & & & $0.62 *$ & 0.35 & 0.20 & -0.325 & 0.51 & 0.20 & -0.11 & -0.01 \\
\hline WIQ: speed & & & & & $0.71^{*}$ & -0.06 & $-0.65^{*}$ & $0.70^{*}$ & 0.06 & -0.43 & -0.04 \\
\hline WIQ: stairs & & & & & & -0.35 & $-0.56^{*}$ & 0.54 & -0.02 & -0.23 & -0.08 \\
\hline \multicolumn{12}{|l|}{ Pain $(\mathrm{P})$} \\
\hline ABI & & & & & & 0.32 & -0.18 & 0.27 & 0.10 & 0.19 & 0.13 \\
\hline WIQ: pain & & & & & & -0.14 & 0.10 & $0.68^{*}$ & 0.09 & 0.03 & -0.27 \\
\hline WIQ: distance & & & & & & 0.24 & -0.15 & 0.41 & 0.13 & -0.07 & 0.04 \\
\hline WIQ: speed & & & & & & 0.09 & -0.53 & $0.71^{*}$ & $0.68^{*}$ & -0.42 & 0.15 \\
\hline WIQ: stairs & & & & & & -0.36 & -0.53 & $0.60^{*}$ & $0.71^{*}$ & -0.29 & -0.03 \\
\hline
\end{tabular}

ADFM p1: ankle dorsiflexion/plantar flexion moment peak 1 during early stance phase. APFM p2: ankle dorsiflexion/plantar flexion moment peak 2 during late stance phase.

KEM p1: knee extension/flexion moment peak 1 during early stance phase.

KFM p2: knee extension/flexion moment peak 2 during late stance phase.

HEM p1: hip extension/flexion moment peak 1 during early stance phase.

HFM p2: hip extension/flexion moment peak 2 during late stance phase.

* Correlation is significant at the 0.05 level (2-tailed).

\subsection{Correlations}

The ABI was found to have a moderate positive correlation with the WIQ subcategory of distance $(r=0.67$; Table 3$)$. During the PF condition, the peak ankle plantar flexor moment had moderate negative correlations with the WIQ subcategories of speed $(r=-0.65)$ and stairs $(r=-0.56)$. However, these correlations became non-significant in the $\mathrm{P}$ condition. Moderate positive correlations were found between the knee extensor moment and the WIQ subcategories of pain $(r=0.71)$ and speed $(r=0.70)$ in the PF condition. In the P condition, an additional moderate positive correlation was found between the WIQ subcategory stairs and the peak knee extension moment $(r=0.60)$. Knee flexion moment was found to have a significant and moderate positive correlation with the WIQ subcategory of pain $(r=0.66)$ during the PF condition, and the WIQ subcategory of speed $(r=0.68)$ as well as the WIQ subcategory of stairs $(r=0.71)$ during the $\mathrm{P}$ condition.

\section{Discussion}

The purpose of this study was to utilize advanced biomechanical analysis to determine the gait impairment of claudicating PAD patients. The present study is the first to provide a detailed quantitative evaluation of the joint kinematic and kinetic changes in PAD patients with bilateral intermittent claudication. Other studies have utilized either just joint kinematics from two-dimensional gait analysis (Crowther et al., 2007) or just ground reaction forces for a kinetic evaluation (Scott-Pandorf et al., 2007), thus providing a limited functional evaluation in gait. Joint kinetics and kinematics were examined while PAD patients walked both with and without claudication pains and were compared to those of gender-, age-, body mass-, and height-matched controls. Our data demonstrate that the gait of claudicating patients is significantly affected both before and after they experience claudication symptoms in their legs. Taken literally, the gait of PAD patients is abnormal from the first step they take. The character of the PAD gait based on our time-distance data appears overall "sluggish and tired" with the majority of the changes becoming most apparent at the level of the hip and ankle joints. The most important findings of this work, however, lie with our joint angle and joint moment data. These data in combination with our time-distance findings suggest that the underlying mechanism in the abnormal PAD gait is a weakness of the hip extensors (gluteal and posterior thigh muscles) and ankle plantar flexors (posterior calf compartment muscles). This weakness appears to be the operating mechanism behind the observed decreased hip extensor and ankle plantar flexor moments. These are then translated at the hip level to decreased hip joint flexion and at the ankle level to increased ankle plantar flexion after heel contact (braking period) and increased ankle dorsiflexion during push off, finally producing the "sluggish and tired" appearing gait of claudicating patients. Gait adaptations, but not ABI, correlated well with all four WIQ subscales indicating that gait impairment quantification via advanced biomechanical analysis may be the most clinically relevant corollary of quality of life deterioration in claudicating patients. ABI did not correlate well with the advanced biomechanical parameters, suggesting that as a hemodynamic index $\mathrm{ABI}$ is unable to capture the complexity of the 
PAD effects on the locomotor pathophysiology of the claudicating limb.

Regarding time-distance parameters, our data demonstrate that compared with healthy controls, PAD patients walk slower, with decreased cadence and decreased stride length, thus spending more time in double support. This all occurs while the PAD patient is walking PF and worsens when the patient experiences claudication leg pain at which time the PAD patient also experiences an increase in stance time and a decrease of the swing phase. Our results are in agreement with those of other studies in PAD patients (Gardner et al., 2001; McDermott et al., 2001) and confirm there is a definite and significant alteration in gait function in patients with PAD at baseline.

Regarding joint kinematics and kinetics, our data demonstrate that compared to healthy controls, PAD patients had reduced peak hip flexion and an increased peak ankle plantar flexion during braking followed by an increased peak ankle dorsiflexion during propulsion. Similarly, compared to healthy controls, PAD patients had reduced peak hip extensor and peak ankle plantar flexor moment. These joint angle and moment changes were present while walking without pain and remained significantly different or even worse with induced pain. The reduced peak hip extensor moment is probably the result of weakness and pain in the extensor muscle group (gluteal and posterior hip compartment muscles). Furthermore, decreased ankle plantar flexor moment suggests weakness of the posterior calf compartment muscles that are unable to adequately propel the claudicating limb. Taken together, these muscle group abnormalities can explain the need claudicants have for shorter steps and spending more time in double support, which in turn results in decreased hip flexion during the braking phase and hip extension during the propulsion phase of walking. The increased ankle dorsiflexion prior to toe off maybe the result of plantar flexor weakness or a mechanism that allows further stretching of the posterior calf compartment muscles in an attempt to assist them in propulsion. Similarly, the initial increased ankle plantar flexion may reflect weakness of the anterior calf compartment muscles that are unable to hold the foot flexed after heel touch down.

Our finding of reduced hip extensor moment is similar to results from elderly populations (Kerrigan et al., 1998; Riley et al., 2001). This result may also explain the contradiction that is found in the literature regarding the hip moment in the elderly, where two studies conducted by Kerrigan et al. (1998) and Riley et al. (2001) have shown deficiencies at the hip for the elderly, while Judge et al. (1996) reported no significant differences at the hip. Because PAD screening has not been performed in these studies, it is possible that the results have been confounded by PAD being present in different subsets of the patients enrolled in these studies. It has been shown that $20-30 \%$ of the elderly population above the age of 65 have PAD in different stages (McDermott, 2002). Therefore, it becomes obvious that appropriate patient screening with exclusion of PAD patients ought to be a necessary step in the design of future gait evaluation studies in the elderly. Decreased plantar flexor moment is a finding observed not only in the elderly (Kerrigan et al., 1998; Riley et al., 2001) but also in disabled patients suffering mostly from osteoarthritis (Kaufman et al., 2001; McGibbon et al., 2001). Reduced plantar flexor function could affect swing initiation (a significant decrease in swing phase has been observed in this study) and trunk progression late in stance (Neptune et al., 2001). Overall, the altered hip and ankle joint kinematics and kinetics we have identified suggest that the propulsion muscles of the leg are weak and fail to support the body in forward progression. This hypothesis is supported by previous findings in our and other laboratories demonstrating PAD patients have significantly decreased propulsion forces (Scott-Pandorf et al., 2007) and ankle plantar flexor strength (Scott-Okafor et al., 2001).

As current guidelines recommend the use of qualitative self-reported measures during studies performed for claudication (Norgren et al., 2007), we evaluated the correlation of advanced biomechanical parameters, ABI and the WIQ subscales. The resting ABI is commonly used as a measure of the hemodynamic compromise seen with PAD (Bauman and Arthur, 1997; Gardner et al., 1997), with a lower ABI value thought to indicate worse claudication effects. ABI did not correlate well with any of the advanced biomechanical parameters, but correlated only with the WIQ subcategory of claudication distance. This finding is in agreement with the results from Izquierdo-Porrera et al. (2005). The above findings continue to underscore that $\mathrm{ABI}$ cannot adequately capture the complexity of the PAD (Green, 2002; McKenna et al., 1991). The small numbers of the significant correlations (5 out of 24 correlations) found between the WIQ subcategories and the joint kinetics may be attributed to the subjective nature of the WIQ evaluation. The small insignificant correlations between the $\mathrm{ABI}$ and the joint kinetics suggest that the clinical ABI measurement may be limited to provide a direct functional evaluation of PAD patients' walking.

Our work points to a weakness in the posterior compartment muscles of the hip and calf as being the key factor underlying the multitude of alterations that characterize PAD gait. Interestingly, this weakness is present before and appears to worsen after the onset of claudication. This finding is consistent with previous reports that demonstrate a muscle metabolic myopathy (Brass and Hiatt, 2000) and an axonal polyneuropathy in the lower extremities of PAD patients (Weber and Ziegler, 2002). Specifically, a number of reports have documented a metabolic myopathy in the PAD muscle that appears to be secondary to defective mitochondrial bioenergetics and related oxidative damage to skeletal muscle structures and components (Pipinos et al., 2006). Mitochondria in PAD muscle have abnormal ultrastructure (Marbini et al., 1986), 
damaged DNA (Bhat et al., 1999), altered enzyme expression and activity, and abnormally high intermediates of oxidative metabolism (Lundgren et al., 1988). Most importantly, evaluations of claudicating muscle mitochondrial bioenergetics demonstrate specific defects in the complexes of electron transport chain with associated compromised mitochondrial respiration (oxidative phosphorylation) and ATP production (Kemp, 2004; Pipinos et al., 2006). This is very similar to those seen in mitochondrial myopathies (Kemp, 2004; Pipinos et al., 2006). Recent work also demonstrates that the mitochondriopathy of PAD muscle is associated with evidence of significant oxidative damage to the muscle components (Pipinos et al., 2006). Furthermore, there is accumulating evidence suggesting that chronic ischemia in PAD patients results in a consistent pattern of electrodiagnostic abnormalities indicating axonal nerve loss (Koopman et al., 1996; Weber and Ziegler, 2002). Therefore, the impairments we have identified at baseline may be reflecting a combination of myopathy and neuropathy in the PAD limbs that becomes worse when exercise-induced ischemia produces progressively worsening ischemic muscle pain and restriction of the lower extremity bioenergetics. The nature of these myopathic and neuropathic changes and the way they are related to the clinical biomechanical findings of leg dysfunction should be the focus of intense future investigation and may hold the key to understanding PAD pathophysiology.

In summary, biomechanical gait analysis indicates significant abnormalities in the gait of claudicating patients. These abnormalities are present both before and after the onset of claudication with several of them becoming worse after claudication onset. Our work points to a weakness in the posterior compartment muscles of the hip and calf as being the key factor underlying the multitude of adaptations that characterize PAD gait. These findings introduce new insights into the pathophysiology of claudicating gait and suggest that biomechanical evaluation may provide a firm foundation for optimal clinical decision-making and assist in an objective measurement of functional outcomes after medical and surgical therapy. For example, vascular surgeons could use the altered gait revealing a specific joint dysfunction of PAD patients to further gain a close scrutinizing procedure about the level of the occlusion prior to a vascular treatment. The altered gait provides a better quantification of the gait abnormality, which subsequently can be addressed surgically to further evaluate gait improvement for the patients by comparing the data before and after treatment. More importantly, the clinicians could use the altered gait to provide a specific joint rehabilitation for PAD patients instead of giving a whole body exercise on treadmill. Thus, biomechanical analysis may facilitate the identification of optimal rehabilitative regimens that could correct the abnormal gait patterns, allowing for greater exercise tolerance.

\section{Conflict of interest}

My coauthors and I have no financial and personal conflicts of interest in relation to the submission, other people and any organization.

\section{Acknowledgments}

We would like to acknowledge the support that was provided by the American Geriatrics Society's Hartford Foundation Dennis W. Jahnigen Award (to JMJ), the Nebraska Research Initiative (to NS), the University of Community on Research and Creative Activity at UNO (to SJC), and the AHA Scientific Councils' student scholarship in cardiovascular disease and stroke (to SAM).

\section{References}

Atkins, L.M., Gardner, A.W., 2004. The relationship between lower extremity functional strength and severity of peripheral arterial disease. Angiology 55, 347-355.

Baker, R., 2006. Gait analysis methods in rehabilitation. Journal of Neuroengineering and Rehabilitation 3, 4.

Barker, S.P., Freedman, W., Hillstrom, H., 2006. A novel method of producing a repetitive dynamic signal to examine reliability and validity of gait analysis systems. Gait \& Posture 24, 448-452.

Basford, J.R., Chou, L.-S., Kaufman, K.R., Brey, R.H., Walker, A., Malec, J.F., Moessner, A.M., Brown, A.W., 2003. An assessment of gait and balance deficits after traumatic brain injury. Archives of Physical Medicine and Rehabilitation 84, 343-349.

Bauman, H.C., Arthur, H.M., 1997. Relationship between functional exercise capacity and general quality of life in nonsurgical patients with lower-extremity peripheral arterial disease. Journal of Vascular Nursing 15, 21-28.

Benedetti, M., Catani, F., Leardini, A., Pignotti, E., Giannini, S., 1998. Data management in gait analysis for clinical applications. Clinical Biomechanics 13, 204-215.

Bhat, H.K., Hiatt, W.R., Hoppel, C.L., Brass, E.P., 1999. Skeletal muscle mitochondrial DNA injury in patients with unilateral peripheral arterial disease. Circulation 99, 807-812.

Brass, E.P., Hiatt, W.R., 2000. Acquired skeletal muscle metabolic myopathy in atherosclerotic peripheral arterial disease. Vascular Medicine 5, 55-59.

Chang, F.M., Seidl, A.J., Muthusamy, K., Meininger, A.K., Carollo, J.J., 2006. Effectiveness of instrumented gait analysis in children with cerebral palsy-comparison of outcomes. Journal of Pediatric Orthopedics 26, 612-616.

Coyne, K.S., Margolis, M.K., Gilchrist, K.A., Grandy, S.P., Hiatt, W.R., Ratchford, A., Revicki, D.A., Weintraub, W.S., Regensteiner, J.G., 2003. Evaluating effects of method of administration on Walking Impairment Questionnaire. Journal of Vascular Surgery 38, 296-304.

Crowther, R.G., Spinks, W.L., Leicht, A.S., Quigley, F., Golledge, J., 2007. Relationship between temporal-spatial gait parameters, gait kinematics, walking performance, exercise capacity, and physical activity level in peripheral arterial disease. Journal of Vascular Surgery $45,1172-1178$.

DiBianco, R., Morganroth, J., Freitag, J.A., Ronan Jr., J.A., Lindgren, K.M., Donohue, D.J., Larca, L.J., Chadda, K.D., Olukotun, A.Y., 1984. Effects of nadolol on the spontaneous and exercise-provoked heart rate of patients with chronic atrial fibrillation receiving stable dosages of digoxin. American Heart Journal 108, 1121-1127.

Fosang, A., Baker, R., 2006. A method for comparing manual muscle strength measurements with joint moments during walking. Gait \& Posture 24, 406-411. 
Gardner, A.W., Clancy, R.J., 2006. The relationship between anklebrachial index and leisure-time physical activity in patients with intermittent claudication. Angiology 57, 539-545.

Gardner, A.W., Sieminski, D.J., Montgomery, P.S., 1997. Physical activity is related to ankle/brachial index in subjects without peripheral arterial occlusive disease. Angiology 48, 883-891.

Gardner, A.W., Forrester, L., Smith, G.V., 2001. Altered gait profile in subjects with peripheral arterial disease. Vascular Medicine 6, 31-34.

Georgoulis, A.D., Ristanis, S., Chouliaras, V., Moraiti, C., Stergiou, N., 2007. Tibial rotation is not restored after ACL reconstruction with a hamstring graft. Clinical Orthopaedics and Related Research 454, 89-94.

Green, S., 2002. Haemodynamic limitations and exercise performance in peripheral arterial disease. Clinical Physiology and Functional Imaging 22, 81-91.

Houck, J., Yack, H.J., Cuddeford, T., 2004. Validity and comparisons of tibiofemoral orientations and displacement using a femoral tracking device during early to mid stance of walking. Gait \& Posture 19, 76-84.

Izquierdo-Porrera, A.M., Gardner, A.W., Bradham, D.D., Montgomery, P.S., Sorkin, J.D., Powell, C.C., Katzel, L.I., 2005. Relationship between objective measures of peripheral arterial disease severity to self-reported quality of life in older adults with intermittent claudication. Journal of Vascular Surgery 41, 625-630.

Jöbges, M., Heuschkel, G., Pretzel, C., Illhardt, C., Renner, C., Hummelsheim, H., 2004. Repetitive training of compensatory steps: a therapeutic approach for postural instability in Parkinson's disease. Journal of Neurology, Neurosurgery, and Psychiatry 75, 1682-1687.

Judge, J.O., Davis 3rd, R.B., Ounpuu, S., 1996. Step length reductions in advanced age: the role of ankle and hip kinetics. The Journals of Gerontology 51, M303-312.

Kaufman, K.R., Hughes, C., Morrey, B.F., Morrey, M., An, K.-N., 2001. Gait characteristics of patients with knee osteoarthritis. Journal of Biomechanics 34, 907-915.

Kemp, G.J., 2004. Mitochondrial dysfunction in chronic ischemia and peripheral vascular disease. Mitochondrion 4, 629-640.

Kerrigan, D.C., Todd, M.K., Della Croce, U., Lipsitz, L.A., Collins, J.J., 1998. Biomechanical gait alterations independent of speed in the healthy elderly: evidence for specific limiting impairments. Archives of Physical Medicine and Rehabilitation 79, 317-322.

Koopman, J.P., de Vries, A.C., de Weerd, A.W., 1996. Neuromuscular disorders in patients with intermittent claudication. The European Journal of Surgery 162, 443-446.

Liles, D.R., Kallen, M.A., Petersen, L.A., Bush, R.L., 2006. Quality of life and peripheral arterial disease. The Journal of Surgical Research 136, 294-301.

Lundgren, F., Bennegård, K., Elander, A., Lundholm, K., Scherstén, T., Bylund-Fellenius, A.C., 1988. Substrate exchange in human limb muscle during exercise at reduced blood flow. The American Journal of Physiology 255, H1156-H1164.

Marbini, A., Gemignani, F., Scoditti, U., Rustichelli, P., Bragaglia, M.M., Govoni, E., 1986. Abnormal muscle mitochondria in ischemic claudication. Acta Neurologica Belgica 86, 304-310.

McDermott, M.M., 2002. Peripheral arterial disease: epidemiology and drug therapy. The American Journal of Geriatric Cardiology 11, 258-266.

McDermott, M.M., Ohlmiller, S.M., Liu, K., Guralnik, J.M., Martin, G.J., Pearce, W.H., Greenland, P., 2001. Gait alterations associated with walking impairment in people with peripheral arterial disease with and without intermittent claudication. Journal of the American Geriatrics Society 49, 747-754.
McGibbon, C.A., Puniello, M.S., Krebs, D.E., 2001. Mechanical energy transfer during gait in relation to strength impairment and pathology in elderly women. Clinical Biomechanics 16, 324-333.

McGinley, J.L., Morris, M.E., Greenwood, K.M., Goldie, P.A., Olney, S.J., 2006. Accuracy of clinical observations of push-off during gait after stroke. Archives of Physical Medicine and Rehabilitation 87, 779-785.

McKenna, M., Wolfson, S., Kuller, L., 1991. The ratio of ankle and arm arterial pressure as an independent predictor of mortality. Atherosclerosis 87, 119-128.

Menard, J.R., Smith, H.E., Riebe, D., Braun, C.M., Blissmer, B., Patterson, R.B., 2004. Long-term results of peripheral arterial disease rehabilitation. Journal of Vascular Surgery 39, 1186-1192.

Meru, A.V., Mittra, S., Thyagarajan, B., Chugh, A., 2006. Intermittent claudication: an overview. Atherosclerosis 187, 221-237.

Neptune, R.R., Kautz, S.A., Zajac, F.E., 2001. Contributions of the individual ankle plantar flexors to support, forward progression and swing initiation during walking. Journal of Biomechanics 34, 1387-1398.

Norgren, L., Hiatt, W.R., Harris, K.A., Lammer, J., 2007. TASC II Section $\mathrm{F}$ on revascularization in PAD. Journal of Endovascular Therapy 14, 743-744.

Oka, R.K., Szuba, A., Giacomini, J.C., Cooke, J.P., 2005. A pilot study of L-arginine supplementation on functional capacity in peripheral arterial disease. Vascular Medicine 10, 265-274.

Pipinos, I.I., Judge, A.R., Zhu, Z., Selsby, J.T., Swanson, S.A., Johanning, J.M., Baxter, B.T., Lynch, T.G., Dodd, S.L., 2006. Mitochondrial defects and oxidative damage in patients with peripheral arterial disease. Free Radical Biology \& Medicine 41, 262-269.

Riley, P.O., DellaCroce, U., Kerrigan, D.C., 2001. Effect of age on lower extremity joint moment contributions to gait speed. Gait \& Posture 14, 264-270.

Runge, M., Hunter, G., 2006. Determinants of musculoskeletal frailty and the risk of falls in old age. Journal of Musculoskeletal \& Neuronal Interactions 6, 167-173.

Scott-Okafor, H.R., Silver, K.K., Parker, J., Almy-Albert, T., Gardner, A.W., 2001. Lower extremity strength deficits in peripheral arterial occlusive disease patients with intermittent claudication. Angiology 52, $7-14$.

Scott-Pandorf, M.M., Stergiou, N., Johanning, J.M., Robinson, L., Lynch, T.G., Pipinos, I.I., 2007. Peripheral arterial disease affects ground reaction forces during walking. Journal of Vascular Surgery $46,491-499$.

Stergiou, N., Ristanis, S., Moraiti, C., Georgoulis, A.D., 2007. Tibial rotation in anterior cruciate ligament (ACL)-deficient and ACLreconstructed knees: a theoretical proposition for the development of osteoarthritis. Sports Medicine 37, 601-613.

Vallée, M., McFadyen, B.J., Swaine, B., Doyon, J., Cantin, J.-F., Dumas, D., 2006. Effects of environmental demands on locomotion after traumatic brain injury. Archives of Physical Medicine and Rehabilitation $87,806-813$.

Weber, F., Ziegler, A., 2002. Axonal neuropathy in chronic peripheral arterial occlusive disease. Muscle \& Nerve 26, 471-476.

Winter, D.A., 2005. Kinetics: forces and moments of force. In: Winter, D. (Ed.), Biomechanics and Motor Control of Human Movement, third ed. Wiley, New York, pp. 86-117.

Yavuzer, G., Eser, F., Karakus, D., Karaoglan, B., Stam, H.J., 2006. The effects of balance training on gait late after stroke: a randomized controlled trial. Clinical Rehabilitation 20, 960-969. 